\title{
Transformaciones en el sistema de salud en Cuba y estrategias actuales para su consolidación y sostenibilidad
}

\author{
Roberto Morales Ojeda, ${ }^{1}$ Pedro Mas Bermejo, ${ }^{2}$ Pastor Castell-Florit Serrate, ${ }^{3}$ \\ Carmen Arocha Mariño, ${ }^{3}$ Nelly C. Valdivia Onega, ${ }^{4}$ Dalilis Druyet Castillo ${ }^{1}$ \\ y José A. Menéndez Bravo ${ }^{1}$
}

Forma de citar Morales Ojeda R, Mas Bermejo P, Castell-Florit Serrate P, Arocha Mariño C, Valdivia Onega NC, Druyet Castillo D, et al. Transformaciones en el sistema de salud en Cuba y estrategias actuales para su consolidación y sostenibilidad. Rev Panam Salud Publica. 2018;42:e25. https://doi.org/10.26633/ RPSP.2018.25

RESUMEN En Cuba, el acceso y la cobertura universales de salud descansan sobre tres principios clave: la salud como derecho humano, la equidad y la solidaridad. Aunque muchos de los indicadores de salud cubanos están entre los mejores de la Región de las Américas, en el 2011 se decidió reorganizar los servicios de salud, a tono con el proceso de actualización del modelo económico y social cubano que transcurría en todos los sectores del país. Para ello, se diseñó un proyecto de investigación-acción que abarcó el diagnóstico de la situación, la implementación de los cambios y la evaluación de los resultados, en varias etapas. Como resultado, se racionalizaron los recursos humanos con una reducción de más de 150000 plazas no vinculadas directamente a la atención del paciente, se aligeraron las estructuras de dirección en 57 municipios, se compactaron 46 policlínicos, se optimizó el Programa del Médico y la Enfermera de la Familia con la proyección comunitaria de 20 especialidades, se reorganizó la docencia y se reordenaron los programas de cooperación médica internacional. Estos cambios han contribuido a mejorar la sostenibilidad del Sistema Nacional de Salud y su desempeño: aumento en el número de consultas en el nivel primario (19,3\%) y estomatológicas $(56,6 \%)$, reducción del número de consultas en cuerpo de guardia $(16,1 \%)$, aumento del número de pacientes intervenidos quirúrgicamente $(12,1 \%)$, incremento del número de proyectos de investigación (300\%) y crecimiento en el número de estudiantes de Medicina (55,7\%), entre otros. El proyecto de transformaciones en la salud emprendido en Cuba continúa.

Palabras clave Sistemas de salud; indicadores de sostenibilidad; eficiencia; Cuba.

La estrategia para el acceso y la cobertura universales de salud descansa sobre tres principios clave: la salud como

\footnotetext{
Ministerio de Salud Pública, La Habana, Cuba.

Instituto de Medicina Tropical Pedro Kourí, La Habana, Cuba. Enviar la correspondencia a Pedro Mas Bermejo, pmasbe@infomed.sld.cu

3 Escuela Nacional de Salud Pública, La Habana, Cuba.

4 Dirección Provincial de Salud de La Habana, Cuba.
}

derecho humano, la equidad y la solidaridad (1). Tanto en el mundo como en la Región de las Américas, la salud universal está ocupando cada vez más el centro de todas las políticas, y los debates se enfocan en las vías que mejor conduzcan a su plena satisfacción. No es ese, sin embargo, el problema de Cuba, que tiene un sistema de salud único a cargo del Estado cubano, con cobertura y acceso sin exclusiones. La sostenibilidad de ese sistema - sin menoscabo de la calidad de la atención- y el alcance de cotas cada vez más altas de eficiencia sí son, sin embargo, problemas apremiantes, a los que se suman - como factores agravanteslos efectos del cambio climático y del envejecimiento poblacional.

Este artículo, que abre el número especial de la Revista Panamericana de Salud

Este es un artículo de acceso abierto distribuido bajo los términos de la licencia Creative Commons Attribution-NonCommercial-NoDerivs 3.0 IGO, que permite su uso, distribución y reproducción en cualquier medio, siempre que el trabajo original se cite de la manera adecuada. No se permiten modificaciones a los artículos ni su uso comercial. Al reproducir un artículo no debe haber ningún indicio de que la OPS o el artículo avalan a una organización o un producto específico. El uso del logo de la OPS no está permitido. Esta leyenda debe conservarse, junto con la URL original del artículo. 
Pública dedicado a la salud en Cuba, tiene el propósito de fundamentar, exponer y analizar la racionalidad de las transformaciones realizadas en el sistema de salud cubano como parte de la estrategia para su reordenamiento y consolidación, y la búsqueda de su sostenibilidad. Con esta información de partida, el lector podrá adentrarse más documentadamente en el resto de los artículos, que abarcan varios planos convergentes: los problemas de salud, sus factores condicionantes y las respuestas del sistema de salud mediante varios de sus programas y estrategias; las consideraciones económicas en torno a la salud pública cubana y su sostenibilidad; y la investigación y las nuevas tecnologías de la información en función de mejorar la calidad y racionalizar el ejercicio de la salud pública en el país, entre otros.

\section{CONSIDERACIONES GENERALES Y ANTECEDENTES}

Un problema crítico para la salud pública mundial emerge de la interrogante siguiente: ¿el Estado debe asumir los servicios de salud, privatizarlos, o aplicar fórmulas mixtas o intermedias? Esta disyuntiva se hizo particularmente apremiante a partir del último decenio del siglo XX con la aplicación de políticas alentadas fundamentalmente por el Banco Mundial y el Fondo Monetario Internacional, que promovían reformas para remodelar los sistemas de salud y reducir la participación del Estado (2). En años más recientes, esta cuestión ha sido objeto permanente de debate y su enfrentamiento varía según el amplio espectro de tendencias políticas y de enfoques económicos (2-6).

Desde el punto de vista de su regionalización y organización político-administrativa, en los sistemas de salud latinoamericanos hay dos tendencias: por un lado, a concentrar los servicios públicos y colectivos dentro de un sistema único y, por otro, a transferir competencias a las autonomías subnacionales. Los casos más antiguos de unificación del sistema de salud son los de Chile, Costa Rica y Brasil, en ese orden. Actualmente avanzan en una dirección similar los de Argentina y Colombia (7-12).

El sistema de salud cubano está organizado por niveles de atención y ofrece una cobertura universal que se aprecia en la atención médica y epidemiológica a la población, sin distinción de color de la piel, credo, ubicación geográfica, o situación económica, social o política (13). La promoción de salud y la prevención de enfermedades son acciones prioritarias. El Sistema Nacional de Salud (SNS) opera bajo las premisas de mejorar los indicadores de salud de la población, la calidad de la atención médica, la satisfacción con los servicios y la eficiencia en el empleo de los recursos para garantizar la sostenibilidad del sistema y su relación con la salud universal (14-16).

A lo largo de los últimos 58 años, y a pesar de las limitaciones materiales, el SNS ha mantenido un perfeccionamiento continuo de sus sistemas y servicios de salud, que le ha permitido mejorar de manera sostenida los principales indicadores sanitarios del país, algo reconocido mundialmente. Cuba ocupa los primeros lugares en varios indicadores clave de salud en la Región $(17,18)$ (cuadro 1) y muestra resultados relevantes en otros indicadores muy sensibles al estado de salud de la población, como los de natalidad y mortalidad (cuadro 2).

Al inicio del siglo XXI, el SNS se encontraba en un proceso de consolidación y renovación. En la primera década se implementó un vasto programa social (19) que generó nuevas acciones de recuperación y modernización de los servicios de salud, con la introducción de tecnología moderna en todos los niveles de atención, incluido el primario. A finales del 2009, durante un discurso ante el Parlamento Cubano, el Presidente Raúl Castro afirmó que "sin afectar a la calidad de la atención de salud que se ofrece a todos los ciudadanos sin costo alguno, e inclusive mejorándola, los gastos pueden reducirse sustancialmente" (20). Esta exhortación, desde el más alto nivel de dirección del país, fue un claro llamado a la eficiencia sin menoscabo de la excelencia en los servicios.

La respuesta del sistema de salud fue la elaboración e implementación de un plan de perfeccionamiento y transformaciones dirigido a reorganizar, compactar y regionalizar los servicios de salud con el fin de consolidar sus acciones e incrementar la eficacia y la eficiencia de los planes y los programas, así como de elevar la capacidad del sistema para enfrentar retos previsibles relacionados con el envejecimiento poblacional y otras contingencias de salud, como las que podrían derivarse del cambio climático y sus consecuencias $(19,21)$.

En el 2011 se inició en Cuba la actualización del modelo económico y social en todos los sectores (22). El Ministerio de Salud Pública, como parte de sus procesos sistemáticos de supervisión y control, se insertó en esa actualización con una evaluación crítica que sacó a la luz un conjunto de dificultades relacionadas con

CUADRO 1. Posición de Cuba con respecto a los países de la Región de las Américas, según indicadores seleccionados, 2015

\begin{tabular}{lrcc}
\hline \multicolumn{1}{c}{ Indicador } & Cuba & $\begin{array}{c}\text { Países de la Región } \\
\text { (mínimo; máximo) }\end{array}$ & $\begin{array}{c}\text { Cuba (posición } \\
\text { relativa en la Región) }\end{array}$ \\
\hline Expectativa de vida (años) & 79,1 & 63,$5 ; 82,2$ & 5 \\
Mortalidad en menores de 5 años (por 1000 nacidos vivos) & 5,5 & 4,$9 ; 69,0$ & 2 \\
Mortalidad materna (por 10 000 nacidos vivos) & 39,0 & $7 ; 359$ & 10 \\
Profesionales de la salud (por 10 000 habitantes) & 157,8 & 7,$5 ; 157,8$ & 1 \\
\hline
\end{tabular}

a Orden de mejor a peor, según la polaridad del indicador.

Fuente: Elaborado por los autores a partir de la referencia 17.

CUADRO 2. Indicadores de natalidad y mortalidad en el mundo, América Latina y el Caribe, y Cuba, 2016

\begin{tabular}{lccc}
\hline \multicolumn{1}{c}{ Indicador } & Mundo & América Latina y el Caribe & Cuba \\
\hline Natalidad & & & 17 \\
$\quad$ Bruta (por 1 000 habitantes) & 19 & 2,1 & 10,4 \\
Fecundidad global (hijos por mujer) & 2,5 & 6 & 8,6 \\
Mortalidad & & 18 & 5,5 \\
Bruta (por 100 000 habitantes) & 8 & 15 & 4,3 \\
Menores de 5 años (por 1 000 nacidos vivos) & 43 & 9 & 2,4 \\
Infantil (por 1 000 nacidos vivos) & 32 & 19 & \\
Neonatal (por 1 000 nacidos vivos)
\end{tabular}

Fuente: Elaborado por los autores a partir de las referencias 17 y 18. 
el funcionamiento de los servicios de salud que requerían transformaciones, así como la necesidad de elaborar una nueva estrategia e implementar líneas de acción dirigidas a reorganizar los servicios, en correspondencia con el contexto económico, social y sanitario del país $(19,23)$.

\section{EL PROYECTO DE TRANSFORMACIONES}

Como consecuencia de la grave crisis económica que sufrió el país en la década de 1990 debido a la pérdida de los mercados tradicionales por los cambios políticos ocurridos en los países de Europa Oriental y el recrudecimiento del bloqueo económico, comercial y financiero impuesto por los Estados Unidos de América contra Cuba vigente desde inicios de la década de 1960 (24), se deterioraron físicamente las unidades de salud, se agravó la carencia de suministros y, en alguna medida, se resintió la calidad de la gestión de salud en los servicios que, a su vez, sufrió un incremento en la demanda de los servicios hospitalarios. En una encuesta realizada por el Instituto Nacional de Higiene, Epidemiología y Microbiología en ese período, se evidenció la insatisfacción de la población con los servicios de salud (25).

En 1997, Suárez (26) mostró la magnitud de los desafíos que el país debería afrontar ante los efectos de la crisis económica para mantener los logros y los niveles de calidad de los servicios en un país con cobertura y acceso universales y gratuitos, con una población acostumbrada a hacer un uso extensivo de esos servicios, desde la atención primaria de salud (APS) hasta los servicios especializados dotados con las tecnologías más avanzadas.

En respuesta a las dificultades detectadas y a la consecuente necesidad de reorganizar los servicios de salud cubanos en armonía con los lineamientos políticos del país y los cambios en el modelo económico que se iniciaron en el 2011 (22), se diseñó un proyecto de investigación-acción. Este proyecto estuvo a cargo de un grupo de expertos conformado por especialistas con más de 5 años de experiencia como directivos en los servicios de salud y con créditos académicos como profesores e investigadores en el área de los sistemas y servicios de salud. El proyecto tuvo dos componentes, uno de diagnóstico y evaluación, y otro de ejecución, implementación y evaluación simultánea de los resultados. El papel principal del grupo de especialistas a lo largo de todo el proceso consistió en el análisis de la información, su discusión colectiva y el asesoramiento técnico a las instancias de dirección del Ministerio de Salud Pública.

\section{Diagnóstico y evaluación}

La primera fase, dedicada al diagnóstico y la evaluación, estribó en la aplicación de un conjunto de 147 indicadores que, según su tipo, miden la estructura, el proceso y los resultados de las instituciones y la satisfacción con los servicios de salud prestados; los resultados se introdujeron en un sistema ad hoc de información de la salud según las diversas categorías (27). Las instituciones que se tomaron en cuenta para el diagnóstico y la evaluación fueron los hospitales, los policlínicos (instituciones de salud que atienden a la población de varios barrios; estos policlínicos están vinculados a hospitales), los consultorios del Programa del Médico y la Enfermera de la Familia (ubicados directamente en cada barrio para atender a la población circundante; están adscritos a los policlínicos), los hogares maternos (centros de atención a las embarazadas con algún tipo de riesgo o que viven en lugares de difícil acceso), los centros y servicios estomatológicos, y las instituciones de atención a las personas mayores o con discapacidad.

Para cada indicador se definió la frecuencia o periodicidad de su recolección (una única vez, mensual, trimestral, semestral o anual), su flujo o niveles de circulación (institucional, local o nacional) y su fuente de captura (estadísticas continuas, encuestas, entrevistas y acciones de supervisión o auditoría). Se precisaron, además, los componentes, la forma de expresión y el algoritmo simple de cálculo de cada indicador. Por su naturaleza, los indicadores podían ser de estructura (necesidad de consultorios para la APS y demanda de recursos humanos), proceso (indicadores generales de recursos y productividad), resultado (cobertura y disponibilidad de los servicios) o satisfacción de la población con los servicios recibidos.

A los fines de la evaluación, se tomaron como base las siguientes definiciones de tres de los conceptos clave en el proceso de transformación (28):

- Reorganización: Proceso mediante el cual se modifica la distribución de las estructuras organizativas para las prestaciones de los servicios, así como los recursos correspondientes, de manera que se pueda dar respuesta a las demandas de salud de la población, en correspondencia con las circunstancias cambiantes y los objetivos individuales y colectivos.

- Compactación: Proceso mediante el cual se fusionan o unifican dos o más secciones o servicios de una misma especialidad o actividad, de forma que garanticen el funcionamiento óptimo con la utilización más racional del tiempo y de los recursos humanos y materiales disponibles.

- Regionalización: Proceso mediante el cual se disponen y organizan los servicios que prestan las instituciones de salud, de forma accesible y equitativa, para garantizar la cobertura a la población según lo requiera, apoyado en el sistema de referencia y contrarreferencia que permite al médico de la familia ser responsable del tránsito de su paciente por el sistema hasta la solución de sus necesidades de salud.

\section{Ejecución, implementación y evaluación de los resultados}

Una vez concluido el componente de diagnóstico y evaluación, cuyo resultado principal fue crear las bases operacionales para modificar la distribución de las estructuras organizativas, y para racionalizar los recursos institucionales e incrementar la eficiencia en la prestación de los servicios — sin menoscabo de la calidad de la atención a las necesidades de la población-, se elaboró un cronograma para la ejecución, implementación y evaluación de los resultados de la transformación. Para ello, se definieron tres etapas, cada una con propósitos y acciones concretas, que se describen a continuación (21). Todo el proceso estuvo a cargo de equipos de profesionales elegidos por su experiencia y competencia específica en dirección y administración de los servicios de salud, en consulta sistemática con las instancias implicadas y la comunidad, con vistas a la toma adecuada de decisiones.

Primera etapa. Realizada en el período 2011-2012, las acciones de esta etapa estuvieron dirigidas a redefinir la plantilla de recursos humanos estrictamente necesaria, aligerar las estructuras de dirección, establecer los consultorios necesarios del Programa del Médico y la Enfermera de 
la Familia, aplicar una nueva estructura en los municipios pequeños con una sola área de salud, reorganizar los procesos docentes y reordenar los programas de cooperación médica internacional.

En esta etapa del proceso de transformaciones se constató que las direcciones municipales de salud y los policlínicos tenían una estructura similar, con independencia de su nivel de complejidad. Se identificaron casos de duplicidad de funciones, pues se habían creado equipos y grupos de trabajo cuyo perfil estaba dirigido a la atención especializada de una enfermedad determinada; esto hacía que los pacientes se atendieran fuera de su consultorio, por lo que se fraccionaba el concepto de responsabilidad y atención continuada. Esta organización asistencial afectaba al cumplimiento de un objetivo fundacional del Programa del Médico y la Enfermera de la Familia: convertir al policlínico y sus consultorios en escenarios asistenciales, docentes e investigativos a partir del proceso de interconsultas con especialistas que transfirieran tecnología al médico de la familia.

Durante esta etapa del proceso de trasformaciones, volvieron al consultorio las acciones de salud que se habían "verticalizado", se incrementó el número de consultorios y se amplió la base del sistema formada por los médicos y el personal de enfermería que atendían a las familias. Con esto, se garantiza la cobertura a la población y se retoma el principio de que la puerta de entrada al sistema es el primer nivel de atención.

Segunda etapa. La evaluación de los resultados de la etapa anterior sentó las bases para una segunda etapa de profundización (2013-2014), en la que se mantuvieron los mismos propósitos y se incluyeron nuevas acciones, a partir de la experiencia adquirida. Entre las nuevas acciones estuvo promover el desarrollo de competencias laborales, éticas y sociales de los trabajadores con el uso de lo más avanzado de la ciencia y la tecnología médicas; además, se fortaleció la organización y la integración entre los diferentes servicios y niveles de atención, y se consolidó el modelo de la medicina familiar.

Tercera etapa. En esta etapa (2015-2016) se ejecutaron acciones para conservar los conceptos fundacionales del Programa del Médico y la Enfermera de la Familia y la calidad de los servicios en los policlínicos. El objetivo fue satisfacer entre $70 \%$ y $80 \%$ de las necesidades de salud de la

CUADRO 3. Desempeño del sistema de salud cubano antes y después de la aplicación de las transformaciones, según indicadores seleccionados

\begin{tabular}{|c|c|c|c|}
\hline Indicador & Antes (2010) & Después (2016) & $\begin{array}{l}\text { Magnitud del } \\
\text { cambio }(\%)\end{array}$ \\
\hline $\begin{array}{l}\text { Consultas en el nivel primario (pacientes atendidos) } \\
\text { (a) }\end{array}$ & 64907659 & 77449154 & 19,3 \\
\hline $\begin{array}{l}\text { Consultas de cuerpo de guardia en los hospitales } \\
\text { (pacientes atendidos) }\end{array}$ & 21788808 & 18286319 & $-16,1$ \\
\hline Cirugías (pacientes atendidos) & 948694 & 1063184 & 12,1 \\
\hline Cesáreas primitivas (porcentaje del total de pacientes atendidas) & 30,5 & 28,3 & $-7,2$ \\
\hline Consultas estomatológicas (pacientes atendidos) & 18649854 & 29213718 & 56,6 \\
\hline Proyectos de investigación & 1183 & 4863 & 311,1 \\
\hline $\begin{array}{l}\text { Matrícula en la carrera de Medicina (número de estudiantes de } \\
\text { pregrado en Medicina, Enfermería y Licenciatura en Tecnología } \\
\text { de la Salud) }\end{array}$ & 32848 & 51152 & 55,7 \\
\hline
\end{tabular}

Fuente: Elaborado por los autores a partir de la referencia 17.

población en el nivel primario de atención; reorganizar los servicios de higiene, epidemiología y microbiología, en correspondencia con la situación nacional e internacional; mantener el principio de la educación en el trabajo durante los procesos docentes, asistenciales e investigativos en las universidades médicas cubanas; elevar el número de las investigaciones operacionales para optimizar la toma de decisiones; incrementar la exportación de los servicios médicos; y diversificar las fuentes de ingresos.

Al comparar algunos indicadores de resultados del crecimiento y la calidad ${ }^{5}$ de los servicios antes y después de la aplicación de las transformaciones, se aprecia una considerable mejoría en el volumen de los servicios prestados, con un aumento en el número de consultas en el nivel primario $(19,3 \%)$ y estomatológicas $(56,6 \%)$, y el número de pacientes intervenidos quirúrgicamente $(12,1 \%)$, entre otros (cuadro 3), aunque esto no pueda atribuirse solo a las transformaciones. Las consultas de cuerpo de guardia en los hospitales disminuyeron en $16,1 \%$, muy posiblemente debido a una mayor capacidad de resolución del nivel primario de atención. Asimismo, se redujo discretamente la proporción de cesáreas primitivas y aumentó notablemente el rendimiento quirúrgico. En las importantes áreas de investigación y docencia se observaron también incrementos notables.

Pasos futuros. El proyecto de transformaciones —con sus componentes de diagnóstico, ejecución, implementación

En Cuba, el aumento de consultas en los policlínicos es también un indicador de adhesión de los pacientes a la APS como puerta de entrada al sistema, que lleva aparejado el decrecimiento de las consultas en los servicios de urgencia hospitalaria; esto hace que se pueda brindar una atención de mayor calidad en los hospitales. y evaluación- no está concluido, pues sus resultados se utilizan para el monitoreo del desempeño del sistema mediante el uso de los recursos y las herramientas analíticas diseñadas para las tres etapas del proyecto.

Se debe resaltar que todo el proceso de transformación del sector de la salud se ha caracterizado por la ejecución de acciones intersectoriales y de integración en todos los niveles -local, intermedio (provincial o regional) y nacional-, lo que ha servido para avanzar en la consolidación de la presencia de los temas prioritarios de la salud en todas las políticas nacionales y sectoriales adoptadas en el país (28).

\section{Otros hitos importantes del proyecto vinculados a las transformaciones}

Entre los hitos importantes del proyecto de transformaciones que apuntan a mejorar la sostenibilidad del sistema, sin afectar al presupuesto dedicado a la salud, se pueden citar:

- El Estado ha mantenido en la última década, una alta prioridad fiscal con porcentajes que, por lo general, sobrepasan el 25\% del total del gasto de la actividad presupuestada (16); por ejemplo, en el período 2012-2015, el gasto dedicado a la salud se ha mantenido por debajo de $10,4 \%$ del producto interno bruto per cápita anual (cuadro 4).

- La nómina de trabajadores del sistema se redujo en más de 150000 plazas en las etapas primera y segunda de las transformaciones. La mayoría de las plazas sujetas al ajuste eran administrativas y de trabajadores no vinculados a la prestación de servicios 
CUADRO 4. Producto interno bruto (PIB) per cápita, y gasto en la salud y la asistencia social. Cuba, 2011-2015

\begin{tabular}{lccc}
\hline & \multirow{2}{*}{$\begin{array}{c}\text { PIB per } \\
\text { cápita }\end{array}$} & \multicolumn{2}{c}{ Gasto total en la salud } \\
\cline { 3 - 4 } Año & $\begin{array}{c}\text { (en pesos } \\
\text { cubanos) }^{\text {a }}\end{array}$ & $\begin{array}{c}\text { En millones de } \\
\text { pesos cubanos }^{\mathrm{a}}\end{array}$ & $\begin{array}{c}\text { Porcentaje } \\
\text { del PIB (\%) }\end{array}$ \\
\hline 2011 & 4367,4 & 5587 & 11,5 \\
2012 & 4498,0 & 4568 & 9,1 \\
2013 & 4614,4 & 5026 & 9,7 \\
2014 & 4649,2 & 5405 & 10,4 \\
2015 & 4849,3 & 5641 & 10,4 \\
\hline
\end{tabular}

a Tasa de cambio: CU $\$ 1,00=$ US $\$ 1,00$, a precios constantes de 1997.

Fuente: Elaborado por los autores a partir de la referencia 30. Todos los datos primarios para la elaboración de este cuadro proceden de las series estadísticas de 1985 a 2015, que se encuentran disponibles en el sitio web de la Oficina Nacional de Estadística e Información de Cuba http://www. one.cu/series2015.htm

a pacientes, ya que en Cuba no sobran ni sobrarán médicos, estomatólogos, enfermeros y tecnólogos de la salud, de acuerdo con la cobertura proyectada de los servicios nacionales y los compromisos internacionales.

- Se racionalizaron las estructuras de dirección en 57 municipios y se compactaron 46 policlínicos que atendían a poblaciones de menos de 5000 habitantes, la cual se redistribuyó en las áreas de salud cercanas para no afectar la cobertura de los servicios. Al equipo básico de salud, integrado a los consultorios del Programa del Médico y la Enfermera de la Familia, se le asignó la atención de una población máxima determinada, lo que amplía su desempeño y responsabilidad con la salud de su población. Este concepto se refuerza mediante las actividades relacionadas con la atención a los ancianos, el asesoramiento genético y la atención a grupos específicos de la población. Además, se complementa con la participación de los especialistas de medicina interna, ginecoobstetricia, pediatría y un licenciado en psicología, que integran el grupo básico de trabajo, y la proyección comunitaria de 20 especialidades, 14 de ellas en los policlínicos y 6 en el municipio; su frecuencia dependerá de la necesidad del territorio, según su cuadro de salud.

- El Programa de Cooperación Médica fue restructurado en tres modalidades: en la primera, Cuba asume los gastos (20 países); en la segunda, los gastos se comparten entre Cuba y el país receptor de la colaboración (17 países); y en la tercera, Cuba recibe ingresos (30 países). En todos los casos se destaca la aplicación del principio de la solidaridad, ya que los profesionales cubanos prestan servicio por lo general en zonas a donde no acuden los profesionales de los países beneficiarios, ya sea por su lejanía, el acceso difícil o el riesgo sanitario. Entre el 2011 y el 2016, período comprendido en el proceso de transformaciones, 140758 profesionales de la salud han brindado servicios en 67 países.

- La restructuración del programa de cooperación médica produjo una tasa de crecimiento anual de los ingresos de más de $200 \%$, a lo cual se suma el monto del ahorro que implicó, en general, la racionalización de los recursos en la prestación de servicios. Todo ello ha permitido disponer de fondos para garantizar la sostenibilidad y el desarrollo del SNS, la adquisición de insumos como medicamentos y reactivos, los programas de inversión, la reparación y el mantenimiento de las instituciones y la introducción de tecnologías médicas, así como elevar el nivel de informatización del sector y mejorar la preparación del personal de la salud en tecnologías avanzadas.

Pese a las nuevas fuentes de financiamiento por los ajustes hechos en el programa de cooperación médica y al incremento de la eficiencia en los servicios de salud, los costos de atención de las enfermedades crónicas, el dinamismo del desarrollo tecnológico, la prevención y atención de las enfermedades emergentes y reemergentes, el envejecimiento poblacional y los efectos del bloqueo económico, comercial y financiero que los Estados Unidos impone al país, constituyen un enorme desafío para la sostenibilidad del SNS. Estos retos deben enfrentarse con la disposición de los profesionales, técnicos y trabajadores; la integración de la comunidad; la aplicación de procesos, innovaciones y estrategias reticulares en la gerencia de los sistemas y los servicios de salud; la capitalización de los recursos humanos; y un mayor empleo de la gestión de la información y el conocimiento.

El mayor dividendo de todo el proyecto de transformaciones va más allá de los resultados expuestos, algunos de los cuales son el efecto directo de las transformaciones, y otros son tendencias que ya estaban en curso y que han concomitado con ellas. Estos beneficios se han materializado a lo largo de todo el proceso, como experiencias generadoras de valiosas enseñanzas (cuadro 5).

\section{CONCLUSIONES}

En correspondencia con la actualización del modelo económico cubano, las transformaciones del sector de la salud han permitido redefinir las funciones y reclasificar las diferentes estructuras y unidades del sistema de salud en los tres niveles de atención, así como la plantilla de recursos humanos necesaria, todo lo cual ha constituido un paso organizativo importante.

Las propuestas para reorganizar, regionalizar y compactar los servicios una vez certificadas las instituciones se aplicaron en todo el país. Esto propició que continuaran mejorando los indicadores de salud de la población cubana, a partir del crecimiento en la actividad de atención de salud, desde los servicios, la promoción y la prevención hasta las actividades de curación y rehabilitación. Todo el proceso de transformación ha transcurrido de forma simultánea y con menos gasto. Los propósitos se han venido cumpliendo y han mejorado los indicadores de proceso y resultado con índices altos de calidad que se deben mantener; el sistema genera más ingresos y continúa desarrollándose, pues todo debe contribuir a la sostenibilidad del SNS. En consecuencia, se ha incrementado la eficiencia en la prestación de los servicios y ha mejorado el desempeño profesional.

Después de la implementación de las transformaciones, se hizo evidente que se podía aumentar la sostenibilidad del SNS, sin dejar de considerar los factores socioeconómicos, culturales y ambientales que representaban desafíos reales para el desarrollo, como el elevado índice de envejecimiento poblacional, la baja tasa de natalidad y el impacto del cambio climático.

\section{RECOMENDACIONES}

Las transformaciones del sistema de salud deben agilizarse para responder a los cambios sociodemográficos del país. El proceso de reducción de gastos debe mantenerse para incrementar la sostenibilidad del sistema y deben continuar la introducción y el uso masivo de la tecnología como sustento de la eSalud, la capacitación y el entrenamiento del capital humano. Se necesita mejorar la capacidad de los sistemas 


\section{CUADRO 5. Lecciones aprendidas durante el proceso de transformaciones en el sector de la salud en Cuba}

\begin{tabular}{|c|c|c|}
\hline Dimensiones & Dificultades & Acciones \\
\hline 1. Estructura & $\begin{array}{l}\text { Se constató que las estructuras administrativas no propiciaban un } \\
\text { mayor vínculo entre los directivos con los sistemas y los servicios } \\
\text { de salud, ni con los pacientes, y la necesidad de incrementar } \\
\text { la capacidad de identificación y solución de los problemas desde } \\
\text { el nivel primario de atención }\end{array}$ & $\begin{array}{l}\text { Se ajustaron las estructuras sobre la base del cumplimiento de las } \\
\text { funciones inherentes a cada cargo, las competencias de sus funcionarios y } \\
\text { el trabajo colaborativo de la salud pública }\end{array}$ \\
\hline $\begin{array}{l}\text { 2. Competencia de los } \\
\text { directivos, profesionales } \\
\text { y técnicos de la salud }\end{array}$ & $\begin{array}{l}\text { Se había reducido la capacidad resolutiva en las decisiones } \\
\text { administrativas, científicas y asistenciales, algo imprescindible } \\
\text { para alcanzar una mejor apreciación de la utilidad de la } \\
\text { investigación —en particular de la investigación operacional bajo } \\
\text { el método de investigación-acción y la evaluativa—como recurso } \\
\text { para el desarrollo del SNS }\end{array}$ & $\begin{array}{l}\text { Se recuperaron espacios formativos, de capacitación y de superación } \\
\text { permanente, que contribuyeron al incremento de las competencias } \\
\text { profesionales e investigativas, entre estos: } \\
\text { la especialidad en administración de salud } \\
\text { otras formas docentes de pre- y posgrado para la calificación técnica } \\
\text { la calificación científica de los profesionales }\end{array}$ \\
\hline 3. Eficiencia del SNS & $\begin{array}{l}\text { No se habían identificado directamente en los escenarios de } \\
\text { trabajo las posibilidades concretas de incrementar la } \\
\text { sostenibilidad del sistema de salud, para lo que se requería la } \\
\text { eficiencia de sus procesos, y el incremento de los servicios, las } \\
\text { tecnologías y las competencias de sus profesionales }\end{array}$ & $\begin{array}{l}\text { Se incrementaron las competencias y el desempeño profesionales } \\
\text { Se implementó el Programa por la Eficiencia } \\
\text { Se racionalizó el uso de las tecnologías mediante una aplicación más } \\
\text { consecuente del enfoque clínico-epidemiológico y con ello se mejoró } \\
\text { la relación médico-paciente y la calidad de los servicios } \\
\text { Se instituyeron procesos de acreditación de los servicios de salud y de los } \\
\text { escenarios docentes }\end{array}$ \\
\hline $\begin{array}{l}\text { 4. Primer nivel de atención } \\
\text { de salud }\end{array}$ & $\begin{array}{l}\text { El proceso de perfeccionamiento de la APS no era constante y se } \\
\text { requería garantizar la resolución y la eficiencia de la atención en el } \\
\text { nivel primario en más del } 70 \% \text { de sus problemas; esto se reflejaba } \\
\text { en el resto de los niveles de atención }\end{array}$ & $\begin{array}{l}\text { Se incrementó el desarrollo integral del Programa del Médico y la } \\
\text { Enfermera de la Familia como el elemento clave de este proceso } \\
\text { Se introdujeron tecnologías de avanzada que contribuyeron al acercamiento } \\
\text { de servicios especializados a la APS } \\
\text { Se comenzó a aplicar la estrategia de informatización del sector, } \\
\text { que sustenta las áreas definidas para la eSalud }\end{array}$ \\
\hline $\begin{array}{l}\text { 5. Segundo nivel de } \\
\text { atención de salud }\end{array}$ & $\begin{array}{l}\text { Problemas en la infraestructura y el equipamiento en los servicios } \\
\text { hospitalarios que requerían de una mejora continua para incrementar } \\
\text { su capacidad de resolución en el control de las principales causas de } \\
\text { muerte y de otros problemas de salud, como complemento de las } \\
\text { acciones del nivel primario de atención }\end{array}$ & $\begin{array}{l}\text { Se introdujeron tecnologías médicas en los servicios secundarios de } \\
\text { acuerdo con la situación de salud } \\
\text { Se mejoró el sistema de referencia y contrarreferencia entre los niveles } \\
\text { de atención primario y secundario }\end{array}$ \\
\hline $\begin{array}{l}\text { 6. Cambios demográficos } \\
\text { y climáticos en el país }\end{array}$ & $\begin{array}{l}\text { El SNS no se hallaba totalmente preparado para el enfrentamiento de: } \\
\text { - la baja fecundidad y natalidad } \\
\text { - el envejecimiento poblacional } \\
\text { - los efectos del cambio climático }\end{array}$ & $\begin{array}{l}\text { Se incrementó la atención a los cambios demográficos relacionados con el } \\
\text { riesgo preconcepcional y la atención a la pareja infértil } \\
\text { Se incluyeron nuevos aspectos en la atención a las personas mayores para } \\
\text { mejorar su calidad de vida con la aplicación de nuevas formas y métodos de } \\
\text { atención, según la cultura y los requerimientos de esta población } \\
\text { Se colabora con otras instituciones en los proyectos para el enfrentamiento } \\
\text { al cambio climático y sus consecuencias }\end{array}$ \\
\hline
\end{tabular}

Nota: SNS: Sistema Nacional de Salud; APS: atención primaria de salud.

Fuente: Elaborado por los autores.

para la captura, el procesamiento, el análisis y la divulgación eficiente de la información a fin de monitorear, evaluar y, eventualmente, medir el impacto de las transformaciones.

Agradecimientos. Los autores agradecen al Ministerio de Salud Pública de

1. Pan American Health Organization, Directing Council. Strategy for universal access to health and universal health coverage. Washington, DC: PAHO; 2014. (CD53/5, Rev. 2). Disponible en: http://www.paho. org/uhexchange/index.php/es/uhexchange-documents/technical-information /26-strategy-for-universal-accessto-health-and-universal-health-coverage / file Acceso el 18 de julio de 2017.

2. Almeida C. Reforma de sistemas de servicios de salud y equidad en América Latina y el Caribe: algunas lecciones de los años 80 y 90. Cad Saude Publica. 2002;18(4):905-25.
Cuba y la Representación de la Organización Panamericana de la Salud / Organización Mundial de la Salud (OPS/OMS) que ofrecieron valiosos aportes en el análisis crítico y los juicios expresados en el presente artículo.

\section{Conflicto de intereses. Ninguno.}

\section{REFERENCIAS}

Disponible en: http://www.scielo.br/scielo.php?pid=S0102-311X2002000400002 \&script=sci_abstract\&tlng=es o http:/ /dx. doi.org/10.1590/S0102-311X2002000400002 Acceso el 18 de julio de 2017.

3. Yamin AE, Frisancho A. Enfoques basados en los derechos humanos para la salud en América Latina. MEDICC Review. 2015;17(supl):5-7. Disponible en: http:/ / www.medicc.org/mediccreview/pdf. php?lang=es\&id=437 Acceso el 17 de julio de 2017.

4. Etienne CF. Achieving universal health coverage is a moral imperative. Lancet. 2014;
Declaración. Las opiniones expresadas en este manuscrito son responsabilidad de los autores y no reflejan necesariamente los criterios ni la política de la Revista Panamericana de Salud Pública/Pan American Journal of Public Health o de la Organización Panamericana de la Salud ni de las instituciones a las que pertenecen los autores.

385. Disponible en: http:/ / www.thelancet. com/journals/lancet/article/PIIS01406736(14)61679-2/fulltext Acceso el 18 de julio de 2017.

5. Finot I. Descentralización en América Latina: teoría y práctica. Santiago: Instituto Latinoamericano y del Caribe de Planificación Económica y Social; 2001. Disponible en: http://repository.un.org/handle/ 11176/358558 Acceso el 18 de julio de 2017.

6. Homedes N, Ugalde A. Las reformas de salud neoliberales en América Latina: una visión crítica a través de dos estudios de caso. Rev Panam Salud Publica. 
2005;17(3):210-20. Disponible en: http:// www.scielosp.org/scielo.php?pid=S1020$49892005000300012 \&$ script $=$ sci_abstract\&tlng=es Acceso el 6 de octubre de 2017.

7. Titelman D. Reformas al sistema de salud en Chile: desafíos pendientes. Santiago: Comisión Económica para América Latina y el Caribe; 2000. Disponible en: http:// repositorio.cepal.org/handle/11362/5081 Acceso el 18 de julio de 2017.

8. Rocha Da Costa R. Descentralização, financiamento e regulação: a reforma do sistema público de saúde no Brasil durante a década de 1990. Red Sociol Polit. 2002;18. Disponible en: http:/ / www.scielo.br/pdf/ rsocp/n18/10702.pdf Acceso el 6 de marzo de 2017.

9. Sojo A. Reformas de gestión en salud en América Latina: los cuasimercados de Colombia, Argentina, Chile y Costa Rica. Santiago: Comisión Económica para América Latina y el Caribe; 2000. Disponible en: https://www.cepal.org/ es/publicaciones /5970-reformas-gestion-salud-america-latina-cuasimercados-colombia-argentina-chile Acceso el 2 de enero de 2018.

10. Castaño R, Arbeláez J, Giedion U, Morales L. Evolución de la equidad en el sistema colombiano de salud. Serie Financiamiento del Desarrollo. Santiago: Comisión Económica para América Latina y el Caribe; 2000. Disponible en: https://www.cepal. org/es/publicaciones/5085-evolucion-laequidad-sistema-colombiano-salud Acceso el 2 de enero de 2018.

11. Labra ME. Las políticas de salud en Chile y Brasil. Apuntes para una comparación. Rev Cienc Soc. 2000;6(2):259-74.

12. Tafani R. Reforma al sector salud en Argentina. Santiago: Comisión Económica para América Latina y el Caribe; 1997. Disponible en: http://www.bvsde.paho. org/bvsacd/cd67/lcl1035e.pdf Acceso el 2 de enero de 2018.

13. Domínguez Alonso E, Zacca Peña E. Sistema de Salud de Cuba. Salud Publica Mex. 2011;53(supl 2). Disponible en: http://saludpublica.mx/index.php/spm/article/ view/5038/10029Acceso el 2 de enero de 2018.

14. Reed G. Charting the course to universal health in the Americas: Cristian Morales $\mathrm{PhD}, \mathrm{PAHO} / \mathrm{WHO}$ Representative in Cuba. MEDICC Review. 2016;18(3). Disponible en: http:/ / www.medicc.org/mediccreview / pdf.php?lang=en\&id=545 Acceso el 6 de octubre de 2017.
15. Morales Ojeda R. Las transformaciones del Sistema de Salud Pública cubano. Rev Cubana Salud Publica. 2017;43(4):1. Disponible en: http://www.revsaludpublica. sld.cu/index.php/spu/article/view / 1208/954 Acceso el 11 de octubre de 2017.

16. Gálvez González AM, González López R, Álvarez Muñiz M, Vidal Ledo MJ, Suárez Lugo NC, Vázquez Santiesteban $\mathrm{M}$. Consideraciones económicas sobre la salud pública cubana y su relación con la salud universal. Rev Panam Salud Publica. 2018;42:e28. doi: 10.26633/RPSP.2018.28

17. World Health Organization. World Health Statistics. Monitoring health for the SDGs. World Health Statistics data visualizations dashboard. Geneva: WHO; 2017. Disponible en: http:/ / www.who.int/gho/ publications /world_health_statistics / 2017/dashboard/en/Acceso el 6 de octubre de 2017.

18. Cuba, Ministerio de Salud Pública. Anuario Estadístico de Salud. 2016. La Habana: MINSAP; 2016. Disponible en: http:/ / files. sld.cu/dne / files / 2017/05/ Anuario_ Estad\%C3\%ADstico_de_Salud_e_2016_ edici\%C3\%B3n_2017.pdf Acceso el 6 de octubre de 2017.

19. Íñiguez Rojas L. Aproximación a la evolución de los cambios en los servicios de salud en Cuba. Rev Cubana Salud Publica. 2012;38(1):109-25. Disponible en: http:// scielo.sld.cu/scielo.php?script=sci_arttext\& pid=S0864-34662012000100011\&lng=es\& nrm=iso\&tlng=es Acceso el 6 de octubre de 2017.

20. Castro Ruz R. Discurso pronunciado en la clausura de la Asamblea Nacional del Poder Popular el 20 de diciembre de 2009. La Habana: Empresa de Tecnologías de la Información y Servicios Telemáticos Avanzados (CITMATEL); 2009. Disponible en: http:/ / www.cuba.cu/gobierno/rauldiscursos/2009/esp/r201209e.html Acceso el 6 de octubre de 2017.

21. Cuba, Ministerio de Salud Pública. Transformaciones necesarias en el Sistema de Salud Pública. La Habana: MINSAP; 2010. Disponible en: http://files.sld.cu/ editorhome/files/2010/11/transformaciones-necesarias-salud-publica.pdf Acceso el 6 de octubre de 2017.

22. Partido Comunista de Cuba. Actualización de los Lineamientos de la Política Económica y Social del Partido y la Revolución para el período 2016-2021. La Habana: Editora Política; 2016. Disponible en: http://www.granma.cu/file/pdf/gaceta/
01Folleto.Lineamientos-4.pdf Acceso el 22 de septiembre de 2017.

23. Fernández Estrada O. El modelo de funcionamiento económico en Cuba y sus transformaciones. Seis ejes articuladores para su análisis. Madrid: Observatorio de la Economía Latinoamericana; 2011. Disponible en: http://www.eumed.net/ cursecon/ecolat/cu/2011/ofe.html Acceso el 6 de octubre de 2017.

24. American Association for World Health. Denial of food and medicine: impact of the US embargo on health and nutrition in Cuba. Washington, DC: AAWH; 1997. Disponible en: http://medicc.org/ns/documents/The_impact_of_the_U.S. Embargo_on_Health_\&_Nutrition_in_ Cuba.pdf Acceso el 2 de enero de 2018.

25. Chang M, Alemán ML, Cañizares MP, Ibarra AM. Satisfacción de los pacientes con la atención médica. Rev Cubana Med Gen Int. 1999:15:541-7. Disponible en: http:/ / scielo.sld.cu/scielo.php?script=sci arttext\&pid=S0864-21251999000500009 Acceso el 6 de octubre de 2017.

26. Suárez Jiménez J. El sistema de salud en Cuba. Desafíos hacia el año 2000. Rev Cubana Salud Publica. 1997;23:5-16. Disponible en: http://scielo.sld.cu/scielo. php? script $=$ sci_arttext \& pid $=$ S086434661997000100001 Acceso el 6 de octubre de 2017.

27. Arocha Mariño C, Vidal Ledo MJ, Pría Barros MC, García Fariñas A, Gómez de Haz H, Presno Labrador C. Indicadores para medir las transformaciones del Sistema Nacional de Salud. Rev Infodir. 2011;13. Disponible en: http://www.revinfodir.sld. $\mathrm{cu} /$ index.php/infodir/article/view / 435/516 Acceso el 6 de octubre de 2017.

28. Castell-Florit Serrate P, Mas Bermejo P. Cuba: time to expand health in all policies. MEDICC Review. 2016;18(4). Disponible en: http:/ /dx.doi.org/10.1590/medicc.2016. 18400011 Acceso el 6 de octubre de 2017.

29. Oficina Nacional de Estadística e Información. Panorama económico y social, Cuba 2015. La Habana: ONEI; 2016. Disponible en: http://www.one.cu/panorama2015.htm Acceso el 18 de abril de 2017.

Manuscrito recibido el 11 de mayo de 2017. Aceptado para publicación, tras revisión, el 17 de enero de 2018. 
ABSTRACT

Transformations in the health system in Cuba and current strategies for its consolidation and sustainability

Keywords Health systems; sustainability indicators; efficiency; Cuba.
In Cuba, universal access and health coverage rest on three key principles: health as a human right, equity and solidarity. Although many of the Cuban health indicators are among the best in the Region of the Americas, in 2011 it was decided to reorganize health services, in line with the process of updating the Cuban economic and social model that occurred in all sectors. For this purpose, an action-research project was designed, including a situation diagnosis, implementation of changes and evaluation of the results, in several stages. As a result, human resources were rationalized with a reduction of more than 150000 posts not directly linked to patient care, management structures were reduced in 57 municipalities, 46 polyclinics were compacted, the Family Physician and Nurse Program was optimized with 20 specialties for the community care, teaching was reorganized, and the international medical cooperation programs were revisited. These changes have contributed to improving the sustainability of the National Health System and its performance: increase in the number of consultations at the primary level $(19.3 \%)$ and oral care visits $(56.6 \%)$, reduction in the number of visits to emergency rooms $(16.1 \%)$, increase in the number of patients surgically treated $(12.1 \%)$, increase in the number of research projects $(300 \%)$ and increase in the number of medical students (55.7\%), among others. In Cuba, transformations in health is an ongoing project.

RESUMO Em Cuba, o acesso universal e a cobertura de saúde dependem de três princípios fundamentais: a saúde como direito humano, equidade e solidariedade. Embora muitos dos indicadores de saúde cubanos estejam entre os melhores da Região das Americas, em 2011 foi decidido reorganizar os serviços de saúde, de acordo com o processo de atualização do modelo econômico e social cubano ocorrido em todos os setores do país. Para o efeito, foi elaborado um projeto de pesquisa-ação, que incluiu o diagnóstico da situação, a implementação das mudanças e a avaliação dos resultados, em várias etapas. Como resultado, os recursos humanos foram racionalizados com uma redução de mais de 150000 postos não diretamente ligados ao atendimento ao paciente, as estruturas de manejo foram reduzidas em 57 municípios, 46 policlínicas foram compactadas, o Programa Médico e Enfermeiro da Familia foi otimizado com a projeção para a comunidade de 20 especialidades, o ensino foi reorganizado, e os programas internacionais de cooperação médica foram reordenados. Essas mudanças contribuíram para melhorar a sustentabilidade do Sistema Nacional de Saúde e seu desempenho: aumento do número de consultas no nível primário $(19,3 \%)$ e odontologia $(56,6 \%)$, redução do número de consultas na emergência $(16,1 \%)$, aumento do número de pacientes tratados cirurgicamente $(12,1 \%)$, aumento do número de projetos de pesquisa (300\%) e crescimento do número de estudantes de medicina $(55,7 \%)$, entre outros. O projeto de transformação em saúde realizado em Cuba continua.

Palavras-chave Sistemas de saúde; indicadores de sustentabilidade; eficiência; Cuba. 